\title{
Cardio-oncology: challenges in modern medicine
}

Cardiovascular diseases and cancer and are the main causes for morbidity and mortality in the western world. Progress in the treatment of cancer have greatly improved survival, leading to a dramatic increase of cancer survivors in the next years. Increased survival and age of cancer patients have unmasked the burden of cancer and cancer therapy-associated cardiovascular diseases. Depending on cancer treatment modalities, early cardiovascular toxicity is observed in up to $48 \%$ of patients. Late cardiotoxicity can be found in 30\% of patients many years after cancer treatment. Nearly all structures of the heart may be affected, depending on age and risk factors of patients and intensity and mode of anti-cancer therapy. Cardio-oncology has therefore developed as a distinct subspecialty in cardiology. Major goals in cardio-oncology are risk assessment before therapy to (I) prevent onset of cardiovascular disease, (II) identification of diagnostic pathways to allow an early diagnosis of cardiovascular complications, (III) characterize the extent of morbidity and mortality as induced by cancer therapies, (IV) establish therapeutic approaches to treat cardiotoxicity and (V) follow-up cancer survivors. The cardiologist working within the cardio-oncology team is confronted with a broad spectrum of therapies and combination protocols. We can expect many novel cancer drugs to be approved each year. The rate and extent of cardiovascular complications particularly for these new therapies remains to be evaluated. Cardio-oncology aims to identify cancer therapy-related cardiovascular side-effects and to provide optimum multidisciplinary care for cancer patients. We are in the process of gaining a profound knowledge on underlying pathomechanisms and clinical implications, but standardized recommendations and structural requirements for the treatment of cardio-oncology patients are still limited. In this edition of the Journal, experts in the field shield light on novel aspects and data for the diagnostic and therapy of cancer therapy—induced cardiovascular diseases. They critically review the literature and give recommendation for the daily practice.

\section{Acknowledgements}

None.

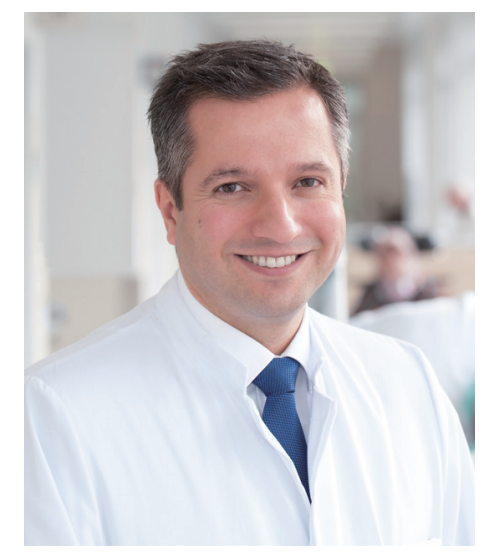

Tienush Rassaf 
Tienush Rassaf, MD

Department of Cardiology and Vascular Medicine, West German Heart and Vascular Center, Medical Faculty, University Hospital Essen, Hufelandstr 55, 45147 Essen, Germany.

(Email:Tienush.Rassaf@uk-essen.de) doi: $10.21037 /$ jtd.2018.12.83

Conflicts of Interest: The author has no conflicts of interest to declare. View this article at: http://dx.doi.org/10.21037/jtd.2018.12.83

Cite this article as: Rassaf T. Cardio-oncology: challenges in modern medicine. J Thorac Dis 2018;10(Suppl 35):S4280S4281. doi: $10.21037 /$ jtd.2018.12.83 